\title{
Implant Rehabilitation Partial Maxillary Edentulism with Onlay Autogenous Chin Bone Grafting and Prosthetic Gingival Restoration: A Case Report
}

\author{
Violeta Malpartida-Carrillo ${ }^{1}$, Pedro L Tinedo-López ${ }^{2}$, Fernando Ortiz-Culca ${ }^{3}$, Alejandro Cornejo-García ${ }^{4}$, Maria E Guerrero ${ }^{5}$, \\ Silvia P Amaya-Pajares ${ }^{6}$
}

\begin{abstract}
Background: Atrophic anterior maxilla rehabilitation can be a challenging procedure due to multiple factors that influence clinical decision making. After a prolonged loss of teeth, the residual bone often impedes the use of standard implant placement protocols and additional procedures are needed.

Aim: The aim of this study is to describe the multidisciplinary approach for the diagnosis and treatment of a 50-year-old woman with prolonged use of a removable maxillary partial denture.

Case Description: This article presents a full-mouth-phased rehabilitation of an atrophic anterior maxilla with three surgical stages. First, onlay autogenous chin bone grafting was used to return the lost tissue. After the consolidation, dental implants were placed in a second stage. A few months later, a connective tissue graft was used to improve the keratinized mucosa width. In the mentioned stages, leukocyte- and platelet-rich fibrin (L-PRF) was used to improve healing and promote tissue regeneration. Finally, prosthetic gingival restoration was used in the anterior region as an alternative to overcome the limitations of hard- and soft-tissue grafting.

Conclusion: The use of autogenous grafts obtained from the chin in combination with xenograft and then covered with an absorbable collagen membrane represents a predictable procedure for the rehabilitation of the long-term partial maxillary edentulism. Prosthetic gingival restoration is an alternative technique to overcome the limitations of hard- and soft-tissue grafting.

Clinical significance: The treatment of a patient with high and width alveolar bone loss needs a multidisciplinary approach. Autogenous grafts obtained from the chin in combination with xenograft and then covered with an absorbable collagen membrane represent an effective procedure. Also, prosthetic gingival restoration can be used as an alternative technique to overcome the limitations of hard- and soft-tissue grafting.

Keywords: Alveolar bone grafting, Alveolar bone loss, Bone regeneration, Dental implants.

The Journal of Contemporary Dental Practice (2019): 10.5005/jp-journals-10024-2610
\end{abstract}

\section{BACKGROUND}

Tooth loss triggers a cascade of biological events with local anatomic changes resulting in both horizontal and vertical reductions that are more prominent in the first year. ${ }^{1,2}$ This condition has been shown to have a direct impact on the patient's quality of life, compromising their ability to masticate, speak, and, in some cases, socialize. ${ }^{3}$ Dental implants are an alternative treatment for missing teeth replacement and its predictability has been confirmed by more than 30 years of experience with high success rates. ${ }^{4,5}$ However, unfavorable local conditions of the alveolar ridge occur due to periodontitis, trauma, tumors, resorption following tooth loss, ${ }^{6}$ oral infections, or long-term edentulism that often compromise the ideal implant placement, and additional procedures are required to improve the bone width and height. Block graft, guided bone regeneration (GBR), distraction osteogenesis, and ridge splitting are procedures used for this purpose ensuring that implants can be placed under optimum conditions. ${ }^{7,8}$

Autogenous block grafting is a reconstructive technique that requires the harvesting of a bone block from an intraoral or extraoral donor site fixing the graft by screws to a recipient site. According to the literature, autogenous block grafting can be considered as the "gold standard" treatment for reconstruction of large horizontal bone defects because of their osteogenic, osteoinductive, and osteoconductive potentials, ${ }^{9-11}$ despite the unpredictable resorption, donor site morbidity, and limited availability. ${ }^{12}$ After
1,2,4,5 Department of Oral Implantology, Faculty of Health Sciences, School of Dentistry, Universidad Cientifica del Sur, Lima, Peru

${ }^{3}$ Department of Stomatology, Faculty of Health Sciences, School of Dentistry, Universidad Cientifica del Sur, Lima, Peru

${ }^{6}$ Department of Restorative Dentistry, School of Dentistry, Oregon Health and Science University, Portland, Oregon, USA

Corresponding Author: Violeta Malpartida-Carrillo, Department of Oral Implantology, Faculty of Health Sciences, School of Dentistry, Universidad Cientifica del Sur, Miraflores, Lima, Peru, Phone: +516106400 (ext 324), e-mail: viletayu_30@hotmail.com

How to cite this article: Malpartida-Carrillo V, Tinedo-López PL, et al. Implant Rehabilitation Partial Maxillary Edentulism with Onlay Autogenous Chin Bone Grafting and Prosthetic Gingival Restoration: A Case Report. J Contemp Dent Pract 2019;20(7):851-856.

Source of support: Nil

Conflict of interest: None

block grafts are fixed, we can use xenografts, allografts, or alloplastic graft materials to fill in the gaps between the recipient site and the block grafts and to minimize block graft resorption. Xenograft is one of the most used graft materials, and, frequently, in the form of an anorganic bovine bone (ABB) can minimize block graft resorption because this osteoconductive material can be placed around block grafts. ${ }^{13}$ Also, ABB acts as a space maintainer and holds the 
space for a longer term since it resorbs very slowly. ${ }^{14}$ Moreover, a membrane is often used to provide structural support to the defect site and to promote the intrinsic regenerative potential of the host tissue. ${ }^{15}$ Hence, bioabsorbable membranes, such as collagen ones, have often been used as tissue barriers for bone augmentation to promote primary wound closure and to minimize graft resorption. ${ }^{16}$ The evidence suggest that in spite of all considerations described above, the resorption occurs., ${ }^{9,16,17}$ Therefore, such resorption may influence the positive impact of grafting on the esthetic outcome. ${ }^{18}$

Tissue engineering has emerged as an alternative technique to repair and restore function of damaged or diseased tissues and its aim is to create bioartificial tissues and organs. ${ }^{19}$ L-PRF is considered one of the growing topics for applied clinical regenerative medicine frequently used in oral and maxillofacial surgery as a surgical adjuvant to improve healing and promote tissue regeneration. ${ }^{20-22}$

Knowing that the treatment of an atrophied alveolar ridge in the anterior zone is acutely complicated, Coachman et al. ${ }^{23}$ suggested a prosthetic gingival restoration as an alternative to overcome the limitations of hard- and soft-tissue grafting. This option can reestablish natural crown ratios and natural gingival profiles, reducing the necessity of additional gingival surgical procedures.

This clinical report describes the multidisciplinary approach for the diagnosis and treatment of a 50-year-old woman with prolonged use of a removable maxillary partial denture. The treatment started with an onlay autogenous chin bone graft followed by the placement of dental implants and rehabilitated with an implant-supported fixed partial prosthesis with a prosthetic gingival restoration.

\section{Case Description}

A 50-year-old woman presented to the dental clinic of the Universidad Cientifica del Sur, Lima, Peru, in good physical and psychological conditions. Her chief complaint was "I feel uncomfortable with my removable maxillary partial denture." Preliminary clinical and radiographic evaluations and dental casts showed an anterior partial maxillary atrophy, as well as a partial edentulism in the mandible. A cone beam-computed tomography (CBCT) showed a horizontal resorption of the alveolar ridge (Fig. 1). The dental diagnosis of the patient included plaque-induced gingivitis, localized moderate chronic periodontitis, partial edentulism, anterior maxillary bone defect class $\mathrm{H}$-m of Wang classification, ${ }^{24}$ and rotated and extruded teeth. According to the preliminary diagnostic wax-up and extraoral evaluation, insufficient restorative space was available so it was decided to increase the occlusal vertical dimension. The patient was informed about the treatment options: inlay/onlay monocortical grafts or GBR and they were presented and discussed. After that, autogenous bone harvesting from the chin area before the insertion of the dental implants was accepted. Finally, the patient signed an informed consent.

\section{First Preimplantology Surgical Stage}

\section{Autogenous Block Grafting}

A preoperative analgesic and anti-inflammatory therapy with ketorolac $30 \mathrm{mg}$ (Corporacion Infamasa S.A., Lima, Peru) and dexamethasone $8 \mathrm{mg}$ (Laboratorios Unidos S.A., Lima, Peru) was administered intramuscularly. Additionally, an antibiotic, antiinflammatory, and analgesic therapy was administered with $2 \mathrm{~g}$ dose of amoxicillin with clavulanic acid (Laboratorios Naturales y Genericos S.A.C., Arequipa, Peru), 1 tablet every 8 hours for 7 days starting on the evening before the surgery, dexamethasone $10 \mathrm{mg}$ (Laboratorios Unidos S.A., Lima, Peru), and ketorolac $10 \mathrm{mg}$ (Corporacion Infamasa S.A., Lima, Peru), 2 times a day for 3 days after surgery was given orally. Oral disinfection was performed before the surgery using $0.12 \%$ chlorhexidine digluconate mouthwash (Perio-Aid, Dentaid S.L., Spain) and it was also recommended after the surgery for 10 days, 2 times per day. The surgical procedure was performed under local anesthesia with lidocaine $2 \%$ with epinephrine 1:80,000 (New Stetic, Antioquia, Colombia). The horizontal incision begins at the distal of canines positioned $2 \mathrm{~mm}$ apically to the mucogingival line and two vertical incisions apically extended provided good access to the mandibular symphysis. This donor site was exposed maintaining $5 \mathrm{~mm}$ safety distance from the mental nerves and from the roots of the teeth. A block size was marked using rotational instruments considering a block thickness of $4 \mathrm{~mm}$ using a periodontal probe (Hufriedy, Chicago, IL, USA). The block was divided into 2 pieces slightly trimmed for better adaptation and they were mobilized manually using surgical chisels and were immediately immersed into the sterile saline solution to prevent dehydration.

The maxillary recipient site was delimited from canine-tocanine. A full-thickness crestal incision was performed continuing into the attached gingiva of the mesial and distal tooth. The lingual and buccal subperiosteal tissues were carefully dissected to gain adequate visibility of the underlying bone. This recipient site was perforated using a number 2 high-speed round bur under copious saline irrigation to increase the blood supply from endosseous vessels and to improve the physical bond between the grafted bone and the recipient site. Later, two osteosynthesis screws (Systex, Curitiba, Brazil) were used to fix the blocks on the recipient site (Fig. 2). A particulate xenograft (Bio-Oss, Geistlich Pharma AG, Wolhusen, Switzerland) was used to fill the voids around the blocks and the recipient site. A resorbable collagen barrier membrane (Neomem, Citagenix, Sao Paulo, Brazil) was then laid over the entire grafted area to improve the outcome and reduce the surface resorption of the transferred graft. L-PRF was prepared in a table centrifuge at 2,700 rpm for 12 minutes in accordance with the protocol suggested by Dohan et al. ${ }^{25}$ and was used inside the full-thickness flap (Fig. 3). The mentalis muscle and the flaps were repositioned using absorbable 4.0 polyglycolic

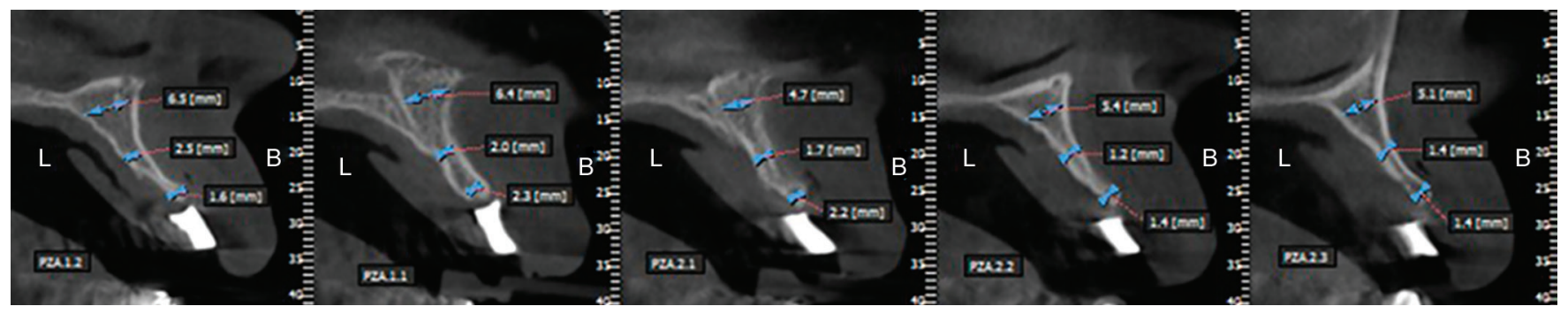

Fig. 1: $C B C T$ showing horizontal resorption of the alveolar ridge 


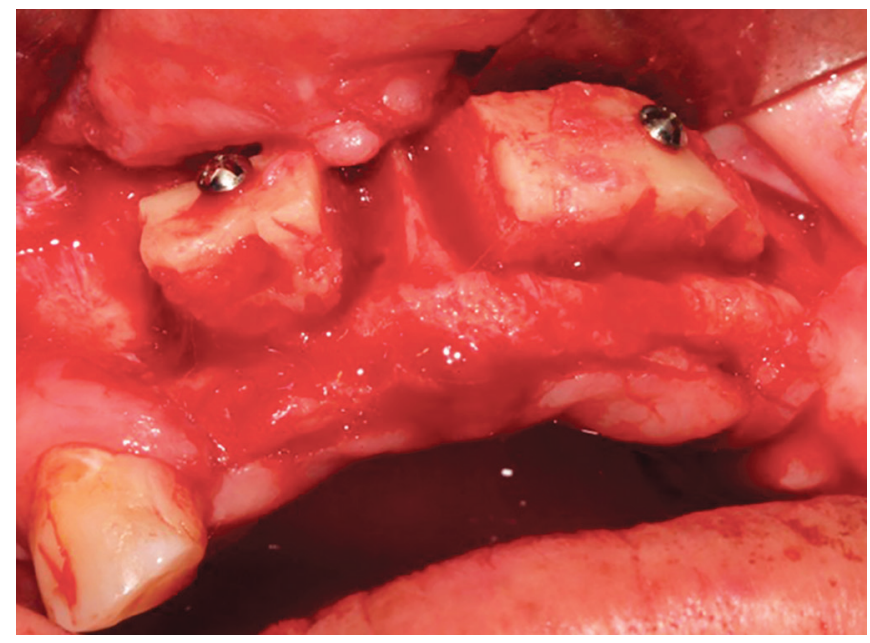

Fig. 2: Two osteosynthesis screws fixing the block to the recipient site

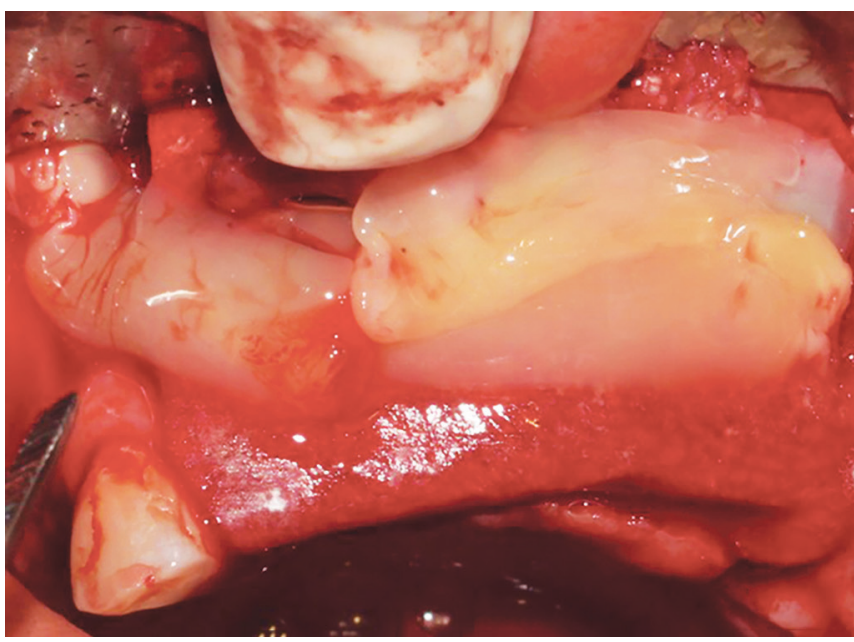

Fig. 3: L-PRF used inside the full-thickness flap

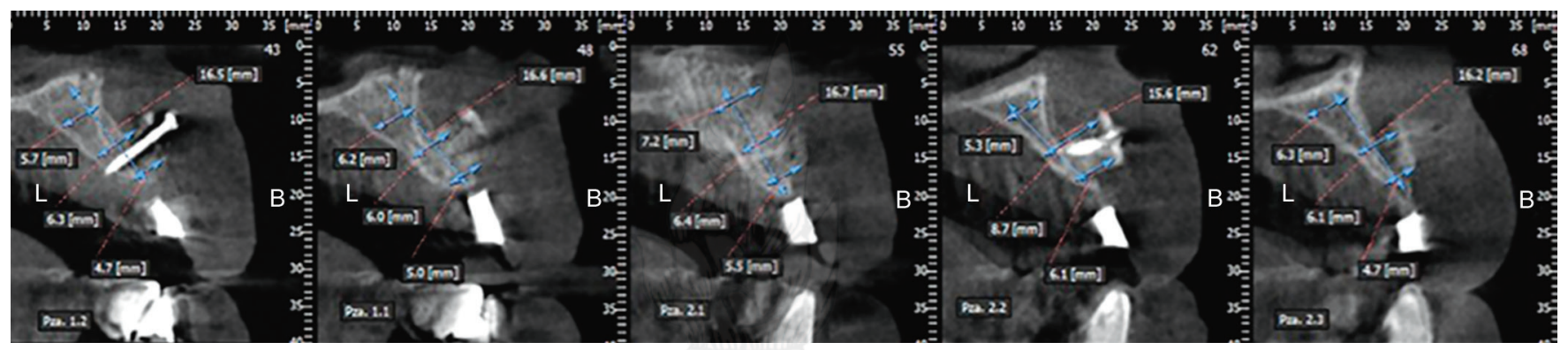

Fig. 4: $C B C T$ showing the horizontal bone gain for implant placement

acid sutures (Unilene S.A.C., Lima, Peru). Postsurgical instructions included a soft diet for 2 weeks and the sutures were removed 14 days postoperatively. Finally, the patient's removable maxillary partial denture was relined with tissue conditioner (Mucopren, Kettenbach, Eschenburg, Germany). The patient had clinical follow-ups every week for the first 3 weeks after surgery and once a month before implant insertion. During this time, the patient's only complaint was the presence of neurosensory disturbance. Vitamin B (Ultra B, Albis S.A., Lima, Peru) therapy was started one tablet every day for 30 days.

\section{Second Surgical Stage \\ Placement of Implants}

Five months after surgery, horizontal bone gain for implant insertion was seen on CBCT planning (Fig. 4). Implants were placed under local anesthesia by the same surgeon who had performed the previous grafting procedure. Preoperative and postoperative analgesic, anti-inflammatory, and antibiotic therapy was prescribed. A full-thickness crestal incision was made and the soft tissues overlying the reconstructed alveolar process were elevated. Four dental implants (IS II Active, NeoBiotech Co, Ltd, Seoul, South Korea) with internal connections $(3.5 \times 10 \mathrm{~mm})$ were placed in the \#12,\#11, \#22, and \#23 teeth regions with GBR, and L-PRF was used inside the full-thickness flap (Fig. 5). Additionally, other 5 implants $(3.5 \times 10 \mathrm{~mm})$ were placed on \#17, \#16, \#15, \#14, and \#24 teeth regions with the same technique. Three mandibular dental implants $(3.5 \times 10 \mathrm{~mm})$ located on the \#36, \#35, and \#46 teeth regions were also placed. The flaps were sutured with absorbable 4.0 polyglycolic acid sutures. The patient's removable maxillary partial denture was relined one more time with a tissue conditioner to avoid tissue pressure. A soft diet and appropriate oral hygiene were recommended for 2 weeks. Sutures were removed 10 days after the surgical procedure. The postoperative recovery was uneventful.

\section{Third Surgical Stage}

Implants Uncovering and Placement of Healing Abutments

Seven months later, a panoramic radiograph was taken to verify the position of the implant. The maxillary and mandibular dental implants were exposed, cover screws were removed, and healing abutments were placed. A connective tissue graft (CTG) with L-PRF was performed to improve the keratinized mucosa width conditioning the peri-implant soft tissues. Sutures were removed after 10 days.

\section{Prosthetic Rehabilitation}

Finally, 4 months after soft-tissue healing and soft-tissue management around maxillary dental implants, the prosthetic stage started with open tray impressions. Cement-retained metalceramic splinted restorations were placed for anterior and posterior maxillary areas. The left mandibular area was rehabilitated with two single screw-retained metal-ceramic crowns and the right side with a cement-retained restoration. Prosthetic gingival restoration was used on the anterior region to improve the natural gingival profiles and the esthetic. Additionally, the crowns of the mandibular anterior region were also replaced (Fig. 6). 


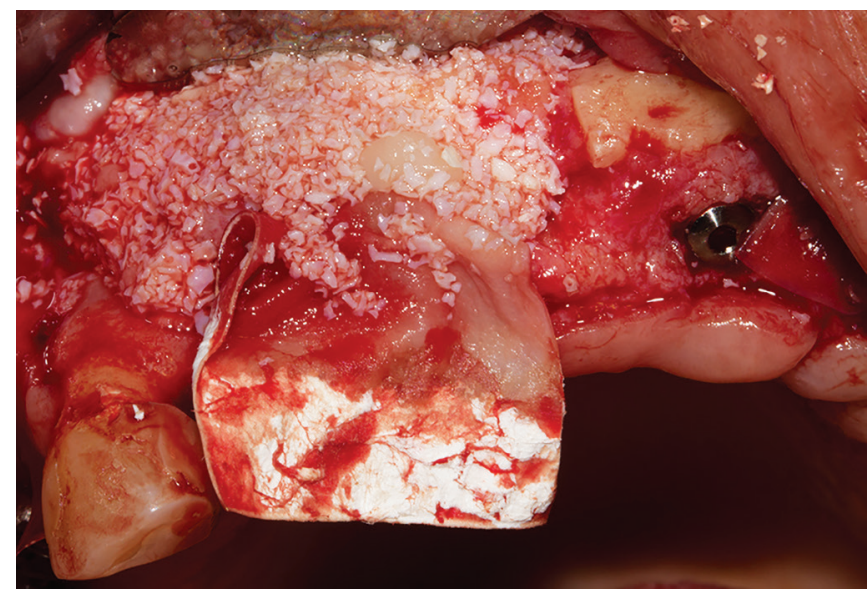

Fig. 5: GBR over the \#12 and \#11 implant sites

\section{Discussion}

A prosthetically driven implant approach recommends that a very deficient edentulous ridge requires alveolar bone reconstruction with different options such as autogenous blocks harvested from intraoral or extraoral donor sites, GBR, ridge splitting, and distraction osteogenesis. ${ }^{7,8}$ The choice of the treatment approach mainly depends of the size and the extension of the defect, medical status of the patient, clinical history of the tooth loss, and the patient's expectations and compliance. ${ }^{26}$

The maxilla is prone to resorption in a centripetal direction; therefore, buccal bone deficiency after tooth loss is constant, leading to complex horizontal deficient ridges. In this case report, the mean width of the remaining alveolar ridge was less than $2 \mathrm{~mm}$ at the middle and coronal regions. Thus, according to the HVC Wang classification, ${ }^{24}$ the dental diagnosis included class $\mathrm{H}-\mathrm{m}$ and the treatment options for the alveolar bone reconstruction in this case were inlay/onlay monocortical grafts or GBR, in the attempt to place the future implants orofacially in a comfort zone. ${ }^{27}$

GBR as well as the use of natural biological molecules (bone morphogenetic proteins and recombinant human platelet-derived growth factors) could have been an alternative. However, we decided to treat this case with autogenous block graft, $A B B$, and collagen membrane because this combination has demonstrated to be an alternative treatment well reported in the literature. ${ }^{12,13,16,28,29}$ However, volume changes due to graft remodeling have been considered one of the main drawbacks of autogenous block grafts. ${ }^{26}$ Chappuis et al. ${ }^{11}$ confirmed a favorable success rate of $98.1 \%$ with a minimal block graft resorption of $7.7 \%$ in a follow-up period of 10 years when autogenous block graft, $A B B$, and collagen membrane were used. Gultekin et al. ${ }^{17}$ evaluated volumetric changes at GBR and autogenous block graft sites in 24 patients using CBCT and they concluded that GBR caused greater resorption at maxillary augmented sites than autogenous block graft. Intraoral block grafts such as chin or mandibular ramus are the preferred sites to obtain predictable sources because they undergo "reverse creeping substitution," which takes longer time for the graft to resorb, hence, they can hold space longer for the bone to fill in. ${ }^{29}$ Ersanli et al. ${ }^{30}$ compared the chin and the ramus efficacy for the restoration of lost horizontal alveolar bone volume in the anterior maxilla in 32 patients, and they had a bone thickness average gain of $4.34 \mathrm{~mm}$ and $4.36 \mathrm{~mm}$, respectively. Both donor sites were a successful option. Donor site morbidity is considered another

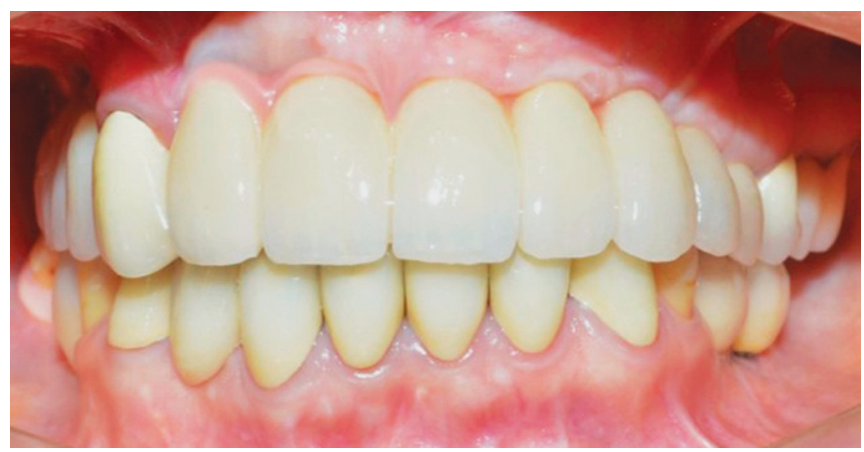

Fig. 6: Final case rehabilitation with prosthetic gingival restoration on the maxillary anterior region

of the autogenous block grafts' drawbacks, mainly related to temporary or permanent neural disturbances involving the inferior alveolar nerve and its branches. ${ }^{31}$ In a subjective and objective cross-sectional evaluation of donor and recipient site up to 4 years, Cordaro et al..$^{32}$ reported alterations of sensitivity in $27.5 \%$ of the 78 patients who received mandibular chin or ramus block grafts with minor complications regarding the donor site area, and the majority of these disturbances were temporary. In this case report after autogenous block grafting, the unique patient's complaint was the presence of neurosensory disturbance; for that, a vitamin B therapy was prescribed, and, after 6 months, the complaint completely disappeared.

Treatment of an atrophied alveolar ridge is extremely challenging because both soft and hard tissues are lost. In this case report, L-PRF was used first inside the full-thickness flap at the GBR procedure and after with CTG in an attempt to create a better environment for bone growth and to enhance soft-tissue healing as reported in the literature. ${ }^{20-22}$ Recently, in a systematic review, Levine et al. ${ }^{33}$ reported that soft-tissue augmentation procedures for mucogingival defects resulted in some significant recession as healing resolved and the tissue matured. Additionally, the area under treatment was extensive. As a matter of fact, before the prosthetic rehabilitation, it was observed that CTG showed a certain tendency to relapse at the \#12 and \#11 teeth regions and it was necessary to recreate the contour of the normal mucosa at that region with a prosthetic gingival restoration. Currently, this procedure is an alternative technique in implant prosthetic treatments for correcting gingival symmetry reducing the necessity of technique-sensitive surgical procedures. ${ }^{23}$ In the literature are reported two common methods of using prosthetic gingival restoration for the management of soft-tissue deficiencies in anterior implant-supported restorations: a crown modified with gingiva-colored porcelain and a customized abutment modified with gingiva-colored porcelain. ${ }^{34}$ The first method is more common to recreate the loss of peri-implant tissue and the second method is used when implants are incorrectly angled or improperly positioned with soft-tissue defects. In this case, was necessary only the applying of gingiva-colored porcelain onto the cervical portion of crowns \#12 and \#11 and the aesthetic result was satisfactory for the patient.

Salama et al. ${ }^{35}$ recommended the inclusion of prosthetic gingival restoration in prosthetic reconstructions as an alternative for aesthetic solutions. The primary issue with this technique is that patients need to understand the importance of adequate oral hygiene habits and their compliance in the maintenance sessions to guarantee the long-term success of their dental 
implants. ${ }^{23,36}$ Therefore, from a practical standpoint, prosthetic gingival restoration is a trustworthy and predictable alternative option adjunct to implant therapy.

\section{ConcLusion}

The use of autogenous grafts obtained from the chin in combination with xenograft and then covered with an absorbable collagen membrane represents an effective and predictable procedure for the rehabilitation of the long-term partial maxillary edentulism. Prosthetic gingival restoration is an alternative technique to overcome the limitations of hard-and soft-tissue grafting, reducing the necessity of additional technique-sensitive surgical procedures.

\section{Clinical Significance}

The treatment of a patient with high and width alveolar bone loss needs a multidisciplinary approach. Autogenous grafts obtained from the chin in combination with xenograft and then covered with an absorbable collagen membrane represent an effective procedure. Also, prosthetic gingival restoration can be used as an alternative technique to overcome the limitations of hard-and soft-tissue grafting.

\section{ACKNOWLedgment}

The authors thank the patient who participated in the study.

\section{References}

1. Schropp L, Wenzel A, et al. Bone healing and soft tissue contour changes following single-tooth extraction: a clinical and radiographic 12-month prospective study. Int J Periodontics Restorative Dent 2003 Aug;23(4):313-323.

2. van der Weijden F, Dell'Acqua F, et al. Alveolar bone dimensional changes of post-extraction sockets in humans: a systematic review. J Clin Periodontol 2009 Dec;36(12):1048-1058. DOI: 10.1111/j.1600051X.2009.01482.x.

3. Gerritsen AE, Allen PF, et al. Tooth loss and oral health-related quality of life: a systematic review and meta-analysis. Health Qual Life Outcomes 2010 Nov;5(6):1-11. DOI: 10.1186/1477-7525-8-126.

4. Sailer I, Mühlemann S, et al. Cemented and screw-retained implant reconstructions: a systematic review of the survival and complication rates. Clin Oral Implants Res 2012 Oct;23(6):163-201. DOI: 10.1111/j.1600-0501.2012.02538.x.

5. Wittneben JG, Millen C, et al. Clinical performance of screw-vs cement-retained fixed implant-supported reconstructions-a systematic review. Int J Oral Maxillofac Implants 2014;29(Suppl):8498. DOI: 10.11607/jomi.2014suppl.g2.1.

6. Shabestari GO, Shayesteh YS, et al. Implant placement in patients with oral bisphosphonate therapy: a case series. Clin Implant Dent Relat Res 2010 Sep;12(3):175-180. DOI: 10.1111/j.1708-8208.2009.00150.x.

7. McAllister BS, Haghighat K. Bone augmentation techniques. J Periodontol 2007 Mar;78(3):377-396. DOI: 10.1902/jop.2007.060048.

8. Chiapasco M, Casentini $P$, et al. Bone augmentation procedures in implant dentistry. Int J Oral Maxillofac Implants 2009;24(Suppl):237259.

9. Lumetti S, Galli C, et al. Correlation between density and resorption of fresh-frozen and autogenous bone grafts. Biomed Res Int 2014 Jun;508328:1-6. DOI: 10.1155/2014/508328.

10. Santana RB, Santana CM. A clinical comparison of guided bone regeneration with platelet-derived growth factor-enhanced bone ceramic vs autogenous bone block grafting. Int J Oral Maxillofac Implants 2015 May;30(3):700-706. DOI: 10.11607/jomi.3529.

11. Chappuis V, Cavusoglu Y, et al. Lateral ridge augmentation using autogenous block grafts and guided bone regeneration: a 10-year prospective case series study. Clin Implant Dent Relat Res 2017 Feb;19(1):85-96. DOI: 10.1111/cid.12438.

12. Jensen SS, Terheyden H. Bone augmentation procedures in localized defects in the alveolar ridge: clinical results with different bone grafts and bone-substitute materials. Int J Oral Maxillofac Implants 2009;24(Suppl):218-236.

13. Cordaro L, Torsello F, et al. Effect of bovine bone and collagen membranes on healing of mandibular bone blocks: a prospective randomized controlled study. Clin Oral Implants Res 2011 Oct;22(10):1145-1150. DOI: 10.1111/j.1600-0501.2010.02093.x.

14. Galindo-Moreno P, Hernández-Cortéz P, et al. Morphological evidences of Bio-Oss ${ }^{\oplus}$ colonization by CD44-positive cells. Clin Oral Implants Res 2014 Mar;25(3):366-371. DOI: 10.1111/clr.12112.

15. Elgali I, Omar O, et al. Guided bone regeneration: materials and biological mechanism revisited. Eur J Oral Sci 2017 Oct;125(5):315-337. DOI: 10.1111/eos.12364.

16. Hernández-Alfaro F, Sancho-Puchades $\mathrm{M}$, et al. Total reconstruction of the atrophic maxilla with intraoral bone grafts and biomaterials: a prospective clinical study with cone beam computed tomography validation. Int J Oral Maxillofac Implants 2013 Jan;28(1):241-251. DOI: 10.11607/jomi.2405.

17. Gultekin BA, Bedeloglu $E$, et al. Comparison of bone resorption rates after intraoral block bone and guided bone regeneration augmentation for the reconstruction of horizontally deficient maxillary alveolar ridges. Biomed Res Int 2016 Oct;4987437:1-9. DOI: 10.1155/2016/4987437.

18. Pieri F, Aldini NN, et al. Esthetic outcome and tissue stability of maxillary anterior single-tooth implants following reconstruction with mandibular block grafts: a 5-year prospective study. Int J Oral Maxillofac Implants 2013 Jan;28(1):270-280. DOI: 10.11607/jomi.2560.

19. Sammartino G, Dohan Ehrenfest DM, et al. Tissue engineering and dental implantology: biomaterials, new technologies, and stem cells. BioMed Res Int 2016 Mar;5713168:1-3. DOI: 10.1155/2016/5713168.

20. Ibraheem W. Effect of platelet-rich fibrin and free gingival graft in the treatment of soft tissue defect preceding implant placement. J Contem Dent Pract 2018 Jul;19(7):895-899. DOI: 10.5005/ jp-journals-10024-2353.

21. Castro $A B$, Meschi N, et al. Regenerative potential of leucocyte- and platelet-rich fibrin. Part A: intra-bony defects, furcation defects and periodontal plastic surgery. A systematic review and meta-analysis. J Clin Periodontol 2017 Jan;44(1):67-82. DOI: 10.1111/jcpe.12643.

22. Castro $A B$, Meschi N, et al. Regenerative potential of leucocyteand platelet-rich fibrin. Part B: sinus floor elevation, alveolar ridge preservation and implant therapy. A systematic review. J Clin Peridontol 2017 Feb;44(2):225-234. DOI: 10.1111/jcpe.12658.

23. Coachman C, Salama M, et al. Prosthetic gingival reconstruction in a fixed partial restoration. Part 1: introduction to artificial gingiva as an alternative therapy. Int J Periodontics Restorative Dent 2009 Oct;29(5):471-477.

24. Wang HL, Al-Shammari K. HVC ridge deficiency classification: a therapeutically oriented classification. Int J Periodontics Restorative Dent 2002 Aug;22(4):335-343.

25. Dohan Ehrenfest DM, Pinto NR, et al. The impact of the centrifuge characteristics and centrifugation protocols on the cells, growth factors, and fibrin architecture of a leukocyte- and platelet-rich fibrin (L-PRF) clot and membrane. Platelets 2018 Mar;24(2):171-184. DOI: 10.1080/09537104.2017.1293812.

26. Doonquah L, Lodenquai R, et al. Surgical techniques for augmentation in the horizontally and vertically compromised alveolus. Dent Clin North Am 2015 Apr;59(2):389-407. DOI: 10.1016/j.cden.2014.10.004.

27. Buser $D$, Martin $W$, et al. Optimizing esthetics for implant restorations in the anterior maxilla: anatomic and surgical considerations. Int J Oral Maxillofac Implants 2004;19(Suppl):43-61.

28. von Arx T, Buser D. Horizontal ridge augmentation using autogenous block grafts and the guided bone regeneration technique with collagen membranes: a clinical study with 42 patients. Clin Oral Implants Res 2006 Aug;17(4):359-366. DOI: 10.1111/j.16000501.2005.01234.x. 
29. Monje A, Monje F, et al. Horizontal bone augmentation using autogenous block grafts and particulated xenograft in the severe atrophic maxillary anterior ridges: a cone-beam computerized tomography case series. J Oral Implantol 2015 Jul;41(4):366-371. DOI: 10.1563/AAID-JOI-D-13-00219.

30. Ersanli S, Arisan V. Evaluation of the autogenous bone block transfer for transfer for dental implant placement: symphysal or ramus harvesting? BMC Oral Health 2016 Jan;26(4):161-168. DOI: 10.1186/ s12903-016-0161-8.

31. Chiapasco M, Zaniboni M, et al. Augmentation procedures for the rehabilitation of deficient edentulous ridges with oral implants. Clin Oral Implants Res 2006 Oct;17(Suppl):136-159. DOI: 10.1111/j.16000501.2006.01357.x.

32. Cordaro L, Torsello F, et al. Mandibular bone harvesting for alveolar reconstruction and implant placement: subjective and objective crosssectional evaluation of donor and recipient site up to 4 years. Clin
Oral Implants Res 2011 Nov;22(11):1320-1326. DOI: 10.1111/j.16000501.2010.02115.x.

33. Levine RA, Huynh-Ba G, et al. Soft tissue augmentation procedures for mucogingival defects in esthetic sites. Int J Oral Maxillofac Implants 2014;29(Suppl):155-185. DOI: 10.11607/jomi.2014suppl.g3.2.

34. Kamalakidis S, Paniz G, et al. Nonsurgical management of soft tissue deficiencies for anterior single implant-supported restorations: a clinical report. J Prosthet Dent 2007 Jan;97(1):1-5. DOI: 10.1016/ j.prosdent.2006.12.005

35. Salama M, Coachman C, et al. Prosthetic gingival reconstruction in the fixed partial restoration. Part 2: diagnosis and treatment planning. Int J Periodontics Restorative Dent 2009 Dec;29(6):572-581.

36. Enríquez $A$, Sánchez $E$, et al. Esthetic restoration with artificial gingiva in an atrophied alveolar ridge: clinical report. Int J Periodontics Restorative Dent 2016 Jul;36(4):567-571. DOI: 10.11607/ prd.2323. 